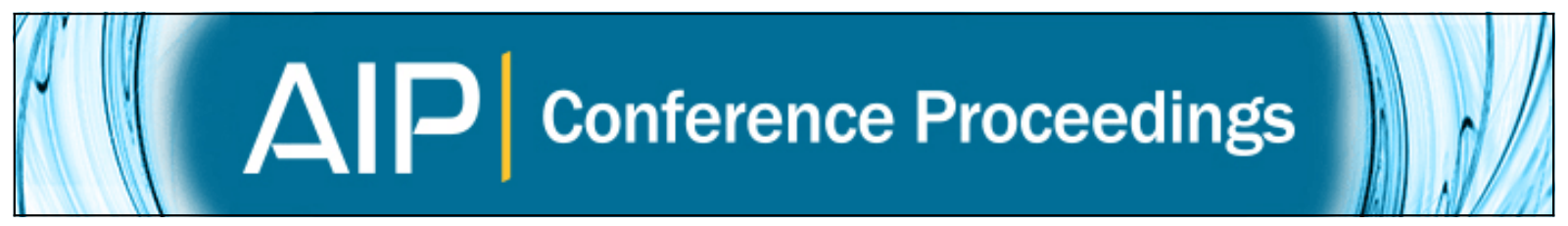

\title{
Weld lines in extrusion: Understanding the role of the flow conditions
}

Olga Sousa Carneiro, Luís Lima Ferrás, Paulo Teixeira, Célio Pinto Fernandes, and João Miguel Nóbrega

Citation: AIP Conference Proceedings 1662, 030012 (2015); doi: 10.1063/1.4918887

View online: http://dx.doi.org/10.1063/1.4918887

View Table of Contents: http://scitation.aip.org/content/aip/proceeding/aipcp/1662?ver=pdfcov

Published by the AIP Publishing

\section{Articles you may be interested in}

The role of initial flow conditions for sibilant fricative production

J. Acoust. Soc. Am. 136, 2922 (2014); 10.1121/1.4900595

Determining The Weld Quality In Extrusion Welding

AIP Conf. Proc. 1353, 461 (2011); 10.1063/1.3589558

The effect of extrusion operating conditions on the on-line apparent viscosity of 98\% Amylopectin (Amioca) and $70 \%$ Amylose (Hylon 7) corn starches during extrusion

J. Rheol. 34, 1245 (1990); 10.1122/1.550085

The role of initial conditions in flow stability with an application to modons

Phys. Fluids 31, 2567 (1988); 10.1063/1.866534

Heat balance and flow conditions for electron beam and laser welding

J. Appl. Phys. 47, 2165 (1976); 10.1063/1.322866 


\title{
Weld Lines in Extrusion: Understanding the Role of the Flow Conditions
}

\author{
Olga Sousa Carneiro ${ }^{1, a)}$, Luís Lima Ferrás ${ }^{1, b)}$, Paulo Teixeira, ${ }^{1, c)}$, \\ Célio Pinto Fernandes ${ }^{1, \text { d) }}$ and João Miguel Nóbrega ${ }^{1, \text { e) }}$ \\ ${ }^{1}$ IPC/I3N - Institute Polymers and Composites, Department of Polymer Engineering University of Minho, \\ Campus de Azurém, 4800-058 Guimarães, Portugal

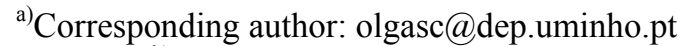 \\ ${ }^{b)}$ luis.ferras@dep.uminho.pt \\ ${ }^{c)}$ p.teixeira@dep.uminho.pt \\ d)cbpf@dep.uminho.pt \\ e)mnobrega@dep.uminho.pt
}

\begin{abstract}
This work presents a numerical and experimental study on the flow behavior of a polymer melt around the spider leg of a prototype extrusion die, designed to study the relationship between the thermo-mechanical conditions in which the weld lines are formed and their impact. The numerical simulations will enable to study the influence of the spider leg location on the flow type developed and on the corresponding stress, velocity and pressure fields. Extrusion runs were also performed in the same conditions and the resulting extrudates were mechanically characterized. These two sources of information (numerical and experimental) are complementary and will, therefore, help to better understand the aforementioned relationship. For the numerical simulations we consider that the flow is isothermal and that the polymer melt (a polystyrene) can be modeled adequately by the multimode Giesekus model.
\end{abstract}

\section{INTRODUCTION}

In polymer processing, weld lines can be formed either by joining two different fluid fronts or when a fluid breaks up due to the presence of an obstacle, and then rejoins downstream. Either way, the processing tool should be designed to minimize the weld lines detrimental effect on the mechanical strength of the final product.

Different mechanisms have been proposed regarding the conditions in which weld lines are formed, justifying the weakness they promote [1-3]. These are: the reorientation of the polymer molecules when the fluid goes through an obstacle, due the existence of an extensional flow (Fig. 1a), the formation of a "skin" layer in the flow front due to exposition of this part of the fluid to different temperatures (Fig. 1b) and the existence of entrapped air at the weld line region (Fig. 1c). In extrusion, the only relevant mechanism is the one illustrated in Fig. 1a, in which the polymer melt that flows close to the spider leg wall, at a very low speed, is abruptly accelerated after detaching from the spider rear end. This acceleration induces molecular orientation along the flow direction and hinders the molecular diffusion that is responsible for the attenuation of the weld line negative effects. 


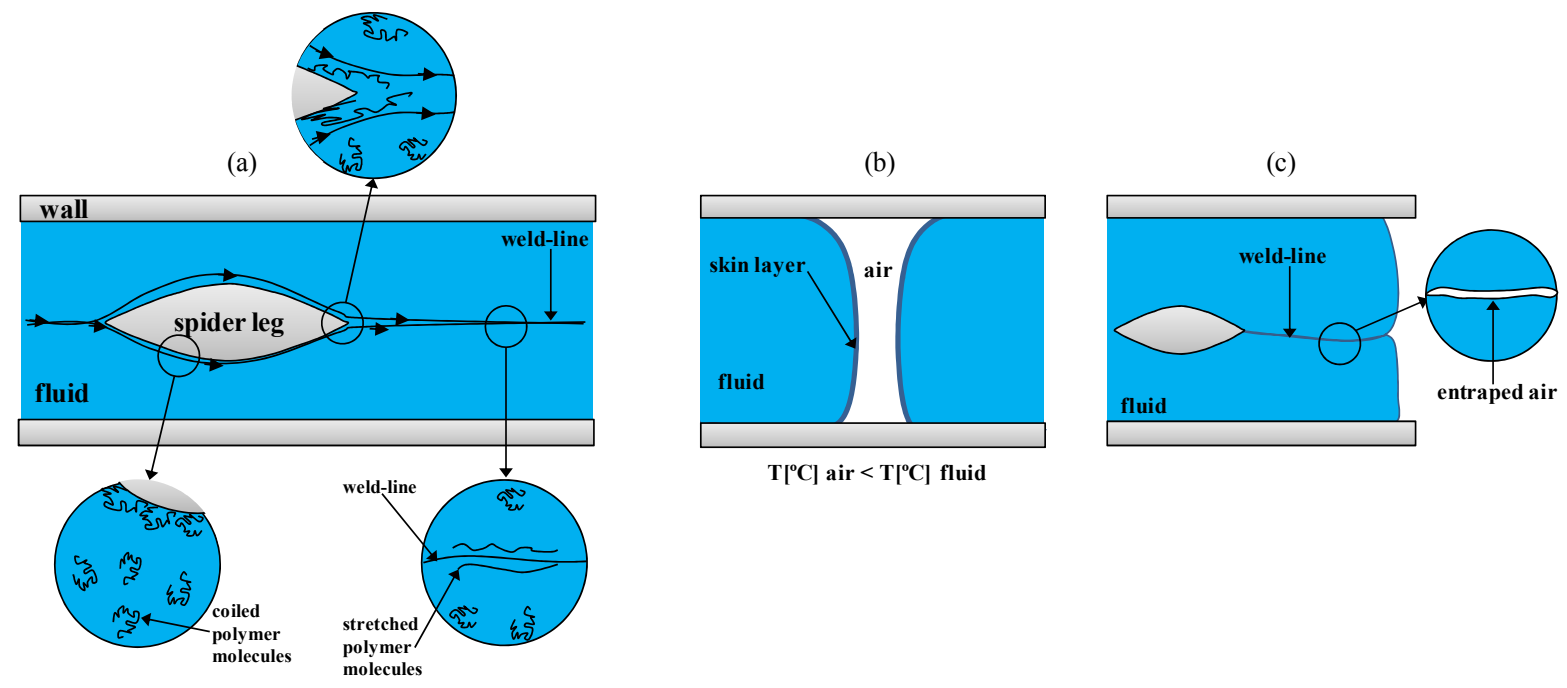

FIGURE 1. Three different mechanisms behind the formation of weld lines.

In extrusion, weld lines are formed in the production of hollow profiles (whose extrusion dies encompass torpedoes supported by spider legs) or when flow separators are employed to promote a better control of the flow distribution [4]. The literature on the formation of weld lines, and their consequences, is more abundant on the area of injection molding [5-12] than on extrusion [13]. Therefore, more studies covering this subject are required in order to improve the insight regarding the mechanism involved in the formation of weld lines in extrusion and, consequently, to provide useful guidelines to circumvent or reduce the material weakness created in the welded regions.

In the literature we can find a few works regarding the numerical simulation of polymer melt flow around spider legs of different shapes [14-17], in which the main conclusions obtained were that the type of flow promoted by the shape of the spider leg has a strong influence on the strength of the weld line it originates. Huang and Prentice [14] showed that for a geometry that does not promote high shear rates at the downstream region of the spider leg, the relative fatigue life of the polymer sample extracted from the weld line region was $90 \%$ of the fatigue life obtained for the remaining regions. However, when using a spider leg geometry similar to the one shown in Fig. 1a, the relative fatigue life was reduced cca 90\%. Later, Mitsuhashi et al. [15-17] presented a remarkable numerical study, extending the work developed by Huang and Prentice to viscoelastic fluids. They showed that by changing only the attack angle of the upstream spider leg region (see Fig. 1a), a great reduction of the extensional dominant flow in that region could be achieved. Their study also included an analysis on the molecular orientation in the weld line region, reinforcing the idea that the type of flow strongly influences the quality of the weld lines, and that a strong extensional flow will align the polymer molecules reducing drastically the capacity of entanglement between molecules of the different flow fronts.

It should be noted that there are some limitations for the design of the spider leg geometry. Depending on the spider leg shape, unwanted secondary flows may be induced, leading to an increase of the residence time of portions of the polymer melt, resulting in material degradation. Thus, some of the geometries proposed by Huang and Prentice [14] are not feasible for application in extrusion dies.

In this work, we combine experimental and numerical approaches to study the effect of the spider leg location (with a specific fixed geometry) on the mechanical properties of extruded rectangular cross section profiles. The data provided by the numerical runs on the type of flow obtained and a detailed study on the evolution and relaxation of stresses and velocity fields, are used to aid the interpretation of the experimental results obtained. For the numerical simulations we use the $n$-mode Giesekus model [18], with the fitting parameters obtained from the rheological characterization of a polystyrene extrusion grade, Polystyrol 158K from BASF. The experimental extrusion runs were performed in the same conditions (i.e., polymer, spider leg location, extrusion temperature and flow rate) of the numerical studies, and the obtained samples were characterized mechanically.

This introduction is followed by Section 2 where the governing equations are presented. In Section 3 we briefly describe the algorithm used to solve the governing equations and present the geometry, the mesh and the flow characteristics. Section 4 is devoted to the rheological characterization of the polymer used to obtain the constitutive 
model parameters. The numerical and experimental results are presented in Section 5, where the main flow features and extrudate mechanical performance are discussed and compared. The paper ends with the main conclusions of the work performed.

\section{GOVERNING EQUATIONS}

The governing equations for confined flow of incompressible fluids are the continuity,

$$
\nabla \cdot \mathbf{u}=0
$$

and the momentum,

$$
\rho \frac{\partial \mathbf{u}}{\partial t}+\rho \nabla \cdot(\mathbf{u u})=-\nabla p+\nabla \cdot \boldsymbol{\tau}
$$

conservation equations, where $\mathbf{u}$ is the velocity vector, $p$ is the pressure, $\rho$ is the fluid density and $\boldsymbol{\tau}=\boldsymbol{\tau}_{\mathrm{s}}+\boldsymbol{\tau}_{\mathrm{p}}$ is the deviatoric stress tensor. The stress tensor is given as a sum of a solvent, $\boldsymbol{\tau}_{s}=\eta_{s}\left(\nabla \mathbf{u}+(\nabla \mathbf{u})^{r}\right)$ (where $\eta_{\mathrm{s}}$ is the solvent viscosity) and a polymer contributions,

$$
\boldsymbol{\tau}_{p}=\sum_{i=1}^{n} \boldsymbol{\tau}_{p i}
$$

which in this case is described by an $n$-mode Giesekus model,

$$
\boldsymbol{\tau}_{p i}+\lambda_{i}\left[\frac{\partial \boldsymbol{\tau}_{p i}}{\partial t}+\mathbf{u} \cdot \nabla \boldsymbol{\tau}_{p i}-\left((\nabla \mathbf{u})^{\mathrm{T}} \cdot \boldsymbol{\tau}_{p i}+\boldsymbol{\tau}_{p i} \cdot \nabla \mathbf{u}\right)\right]+\frac{\alpha_{i} \lambda_{i}}{\eta_{p i}}\left(\boldsymbol{\tau}_{p i} \cdot \boldsymbol{\tau}_{p i}\right)=\eta_{p i}\left(\nabla \mathbf{u}+(\nabla \mathbf{u})^{\mathrm{T}}\right)
$$

where $\lambda$ is the polymer relaxation time, $\alpha$ is the mobility factor, associated with anisotropic Brownian motion and anisotropic hydrodynamic drag of the polymer molecules, and, $\eta_{\mathrm{p}}$ is the polymer viscosity coefficient. The subscript $i$ represents each individual mode used.

\section{NUMERICAL METHOD AND GEOMETRY}

\section{Numerical method}

The system of Eqs. 1, 2, 3 and 4 is solved using a methodology based on the finite volume method, with the help of the extended version of the open-source software OpenFOAM [19]. The PISO (Pressure Implicit with Splitting of Operators) method was used to couple velocity, pressure and stress fields (although it was originally developed for Newtonian fluids [20]). For the numerical solution of the velocity and stress linear systems of equations we used the biconjugate gradient stabilized method; for the pressure, the conjugate gradient method was used together with a GAMG (generalized geometric-algebraic multi-grid solver) preconditioner.

The discretization schemes used are central differences for the diffusive terms and Van-Leer for the convective terms of both momentum and constitutive equations.

\section{Geometry}

The actual geometries of the extrusion die and spider leg are shown in Fig. 2. For the experimental work different (discrete) locations for the spider leg were considered by resorting to a special positioning system encompassing location pins placed along the parallel zone of the extrusion die [21]. 


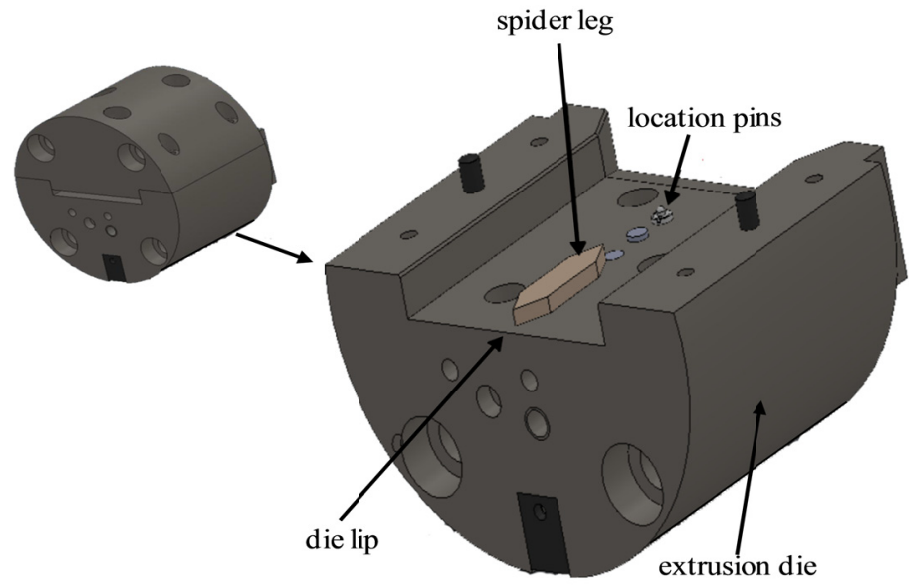

(a)

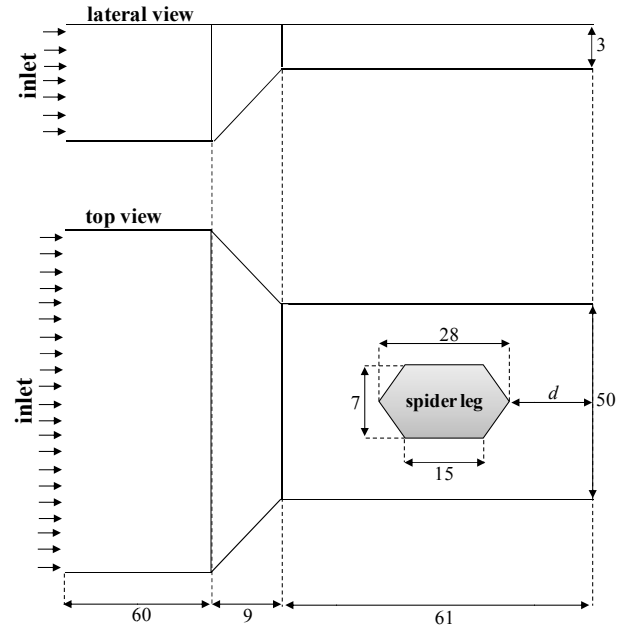

(b)

FIGURE 2. (a) View of the parallel zone of the extrusion die used in the experimental work and (b) corresponding lateral and top views of the geometry used in the numerical simulations (dimensions in $\mathrm{mm}$ ).

Figure 3 shows the computational geometry. The flow channel pre-parallel zone was included to get a nearly fully developed flow upstream the transition zone. The location pins used in the extrusion die determine the places where the spider leg can be placed (in this work three distances $d$ to the exit will be considered: $27\left(d_{1}\right), 19.6\left(d_{2}\right)$ and $\left.12\left(d_{3}\right) \mathrm{mm}\right)$.

A typical mesh used in the simulations is shown in Fig. 3. In order to control the numerical error, three increasingly refined meshes were built, with 34,300; 274,400 and 2,195,200 computational cells. The mesh sensitivity studies performed allowed concluding that the second mesh (Fig. 3) could provide sufficiently accurate results.
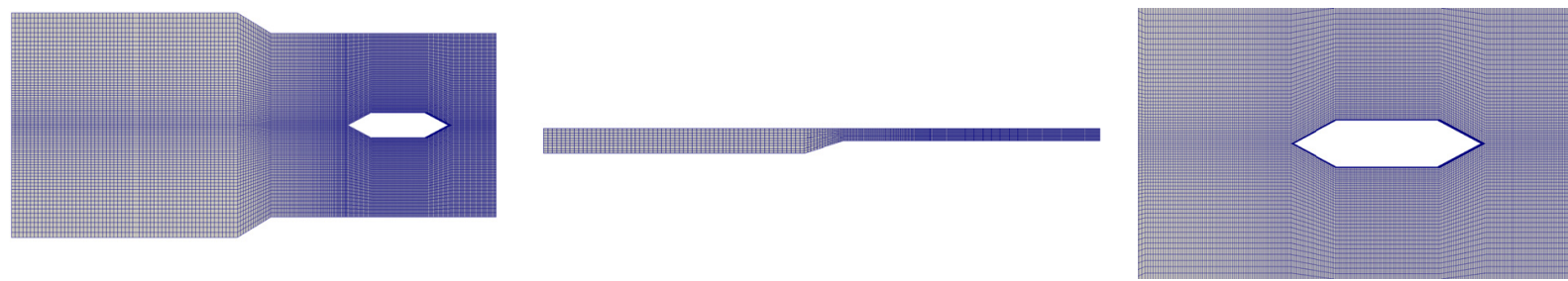

FIGURE 3. Mesh used in the simulations (from left to right: top-view, lateral-view, zoomed view of the spider leg region).

\section{RHEOLOGICAL CHARACTERIZATION}

For the rheological characterization of the polystyrene employed in this study (Polystyrol $158 \mathrm{~K}$, from BASF), parallel-plate and capillary rheometers were used to obtain the viscosity, the loss and the storage modulus (Fig. 4a), at three different temperatures $\left(210^{\circ} \mathrm{C}, 230^{\circ} \mathrm{C}\right.$ and $\left.250^{\circ} \mathrm{C}\right)$. Since the range of temperatures used is small, the shift factor,

$$
a_{T}=\frac{\eta_{0}(T)}{\eta_{0}\left(T_{0}\right)} \frac{T_{0}}{T} \frac{\rho_{0}}{\rho}
$$

was simplified and given by $a_{\mathrm{T}}=\eta_{0}(T) / \eta_{0}\left(T_{0}\right)$, where $\eta_{0}$ stands for the low shear rate Newtonian plateau, obtained with the Carreau-Yasuda model fitting of the shear viscosity, for each temperature, as shown in Fig. 4b. 

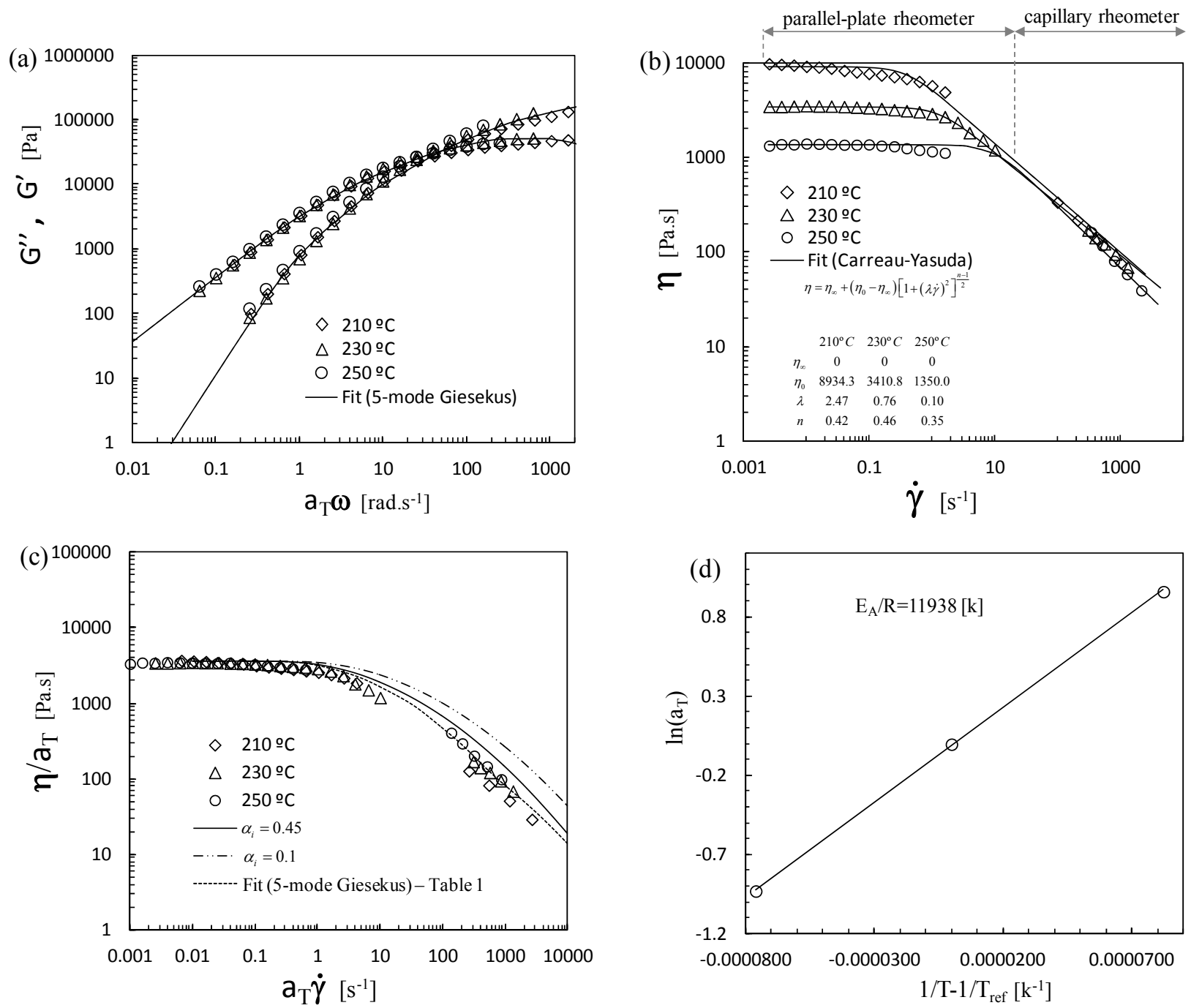

FIGURE 4. Polystyrene rheological characterization and fitted model: (a) $G$ ' and $G$ "' as function of frequency (b) flow curve, (c) master flow curve and (d) shift factor.

Figure 4 shows the fit obtained for $\eta, G^{\prime}$ and $G^{\prime}$, using a 5-mode Giesekus model (see Tab. 1). In Fig. 4a the loss and storage modulus are shown for the three different temperatures tested, using the Time-Temperature superposition principle, illustrating the good agreement obtained between the experimental and the fitted model predictions. In Figs. 4b and c, a fit of the shear viscosity is presented, using the Carreau-Yasuda inelastic model and also the Giesekus model, and Fig. 4d plots the shift factor as function of the temperature. For the constitutive model, the relaxation time $\left(\lambda_{\mathrm{i}}\right)$ and viscosities $\left(\eta_{\mathrm{i}}\right)$ were obtained by fitting the oscillatory shear tests (Fig. $4 \mathrm{a}$ ), while the mobility factors $\left(\alpha_{\mathrm{i}}\right)$ were computed by fitting the flow curve (Fig. $4 \mathrm{c}$ ).

Regarding the shear viscosity data (Fig. 4c), there are some small differences between the experimental data and the theoretical model. One possible reason for that is the fact that we used two different types of rheometers, which have different errors associated, in order to cover the entire shear rate range of interest. Anyway, the shear-thinning region is well modeled with the 5 modes shown in Tab. 1. However, the values obtained for the mobility factors $(\alpha)$ do not provide a typical behavior for the uniaxial extensional viscosity (Fig. 5). This may be a consequence of making the fit with only the shear viscosity, which was the only available for this material, instead of both shear and elongational viscosity. 
TABLE 1. 5-mode Giesekus model parameters.

\begin{tabular}{cccccc}
\hline Mode & $\lambda_{\mathrm{i}}[\mathrm{s}]$ & $\eta_{\mathrm{i}}[\mathrm{Pa}]$ & $\alpha_{\mathrm{i}}[-]$ & $\alpha_{\mathrm{i}}[-]$ & $\alpha_{\mathrm{i}}[-]$ \\
\hline 1 & 0.0041 & 257.5024 & 0.95 & 0.45 & 0.1 \\
2 & 0.0212 & 692.7756 & 0.98 & 0.45 & 0.1 \\
3 & 0.7326 & 1254.4852 & 0.90 & 0.45 & 0.1 \\
4 & 0.0007 & 53.8031 & 0.20 & 0.45 & 0.1 \\
5 & 0.1251 & 1380.1157 & 0.90 & 0.45 & 0.1 \\
\hline
\end{tabular}

Due to the uncertainty of the values employed for $\alpha$, we decided to complement the study of the model fitted experimentally (Tab. 1) with two extreme values usually presented in the literature, 0.1 and 0.45 for all modes (see Fig. 4c and Tab. 1), aiming to evaluate its effect on the results obtained. This information will be useful to evaluate the relevance of improving the material characterization in future works. For illustration purposes, Fig. 5 shows the elongational viscosity for the parameters obtained experimentally (from now on, referred to as Giesekus fit) and the ones with the $\alpha$ extreme values referred above. These curves show a highly extension thickening behavior when $\alpha_{\mathrm{i}}=$ 0.1 and an almost constant elongational viscosity when $\alpha_{i}=0.45$ [22]. For the original fitted model parameters, the predicted elongational viscosity presents an extensional thinning behavior as predicted by Bach et al. [23] for a polystyrene of higher molecular weight than that of the one used in this work. However, the work of Nielsen et al. [24] shows that for PS $50 \mathrm{~K}, 100 \mathrm{~K}$ and $200 \mathrm{~K}$, a small thickening region is obtained. These uncertainties reinforce the purpose of making the study with different values for the mobility parameter. Physically, there should be a thinning region for high elongation rates, but, since we kept constant the mobility parameter (for $\alpha_{\mathrm{i}}=0.45$ and $\alpha_{\mathrm{i}}=0.1$ ), the model cannot predict such behavior.

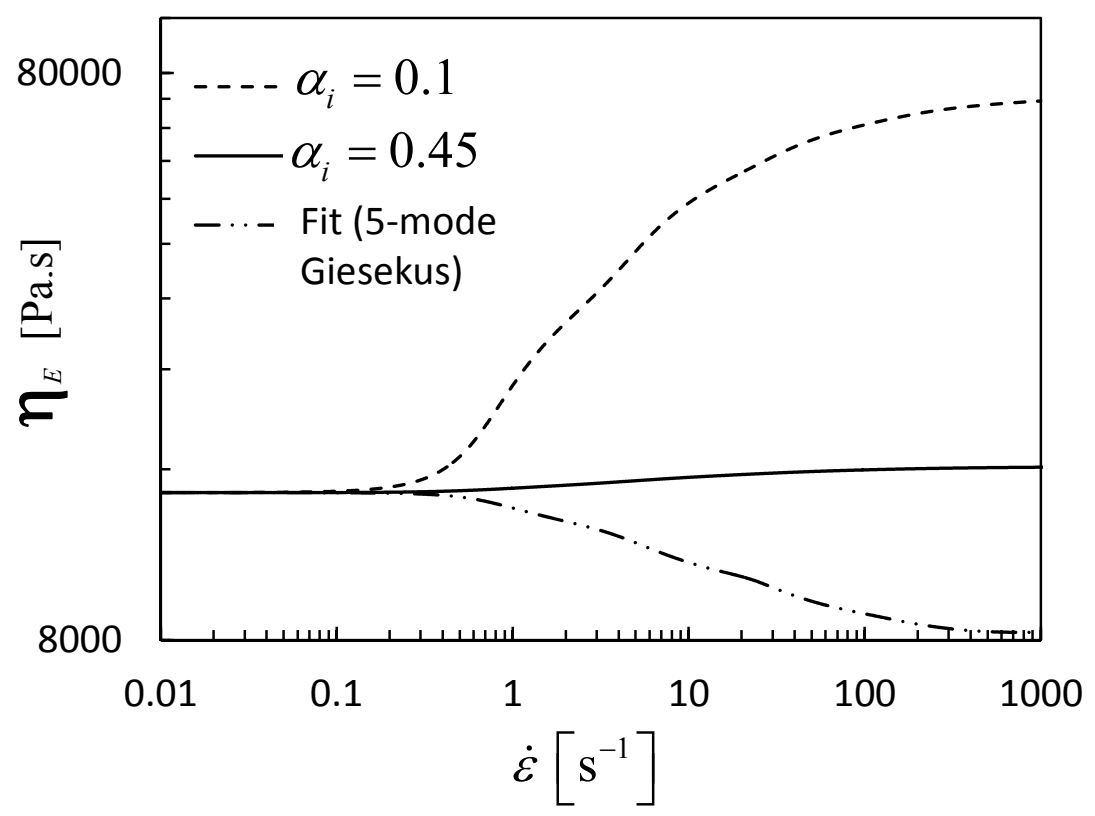

FIGURE 5. Uniaxial elongational viscosity as function of the elongation rate, for three different Giesekus multimode fits.

The experimental setup was tested with mass flow rates of 4 to $6.3 \mathrm{~kg} \cdot \mathrm{h}^{-1}$ ( $U_{\text {outlet }}$ from 0.42 to $0.67 \mathrm{~m} . \mathrm{min}^{-1}$ ), resulting in Deborah numbers (Eq. 5) of 0.716 and 1.127, respectively,

$$
D e=\frac{\lambda_{m} U_{\text {outlet }}}{H_{\text {outlet }}},\left(\lambda_{m}=\frac{\sum_{i} \eta_{i} \lambda_{i}}{\sum_{i} \eta_{i}}\right) .
$$




\section{RESULTS AND DISCUSSION}

The results obtained from the simulations will be presented and described in this section. The discussion will be focused on the type of flow developed around the spider leg and the changes in velocity and stress fields. Finally, the influence of the spider leg location on the type of flow will be studied, combining the numerical and experimental work performed.

In Fig. 6 we can see the distribution of the velocity, and flow type, $\xi$, around the spider leg, for $\alpha_{\mathrm{i}}=0.1$ (for the remaining models the results are very similar). The flow type parameter is given by [25],

$$
\xi=\frac{|\mathbf{D}|+|\Omega|}{|\mathbf{D}|-|\Omega|},
$$

where $|\mathbf{D}|$ and $|\boldsymbol{\Omega}|$ are the magnitudes of the deformation and vorticity tensors, respectively (for a thorough discussion on this subject, the works by R. L. Thomson and P. R. S. Mendes [26] and R. L. Thomson [27] are advised).

As expected, the velocity magnitude increases (due to continuity) when the flow channel is divided by the spider leg (Figs. 6a and b). This division will promote an extensional flow at the location of the flow front merging, as can be seen in Figs. 6c and d.

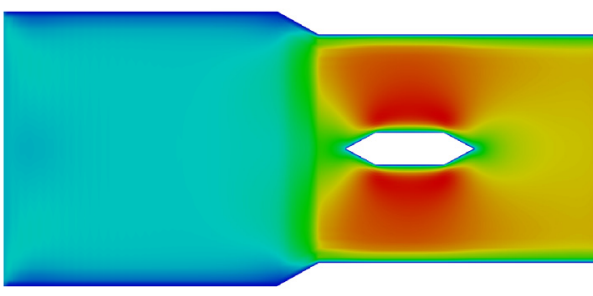

(a)

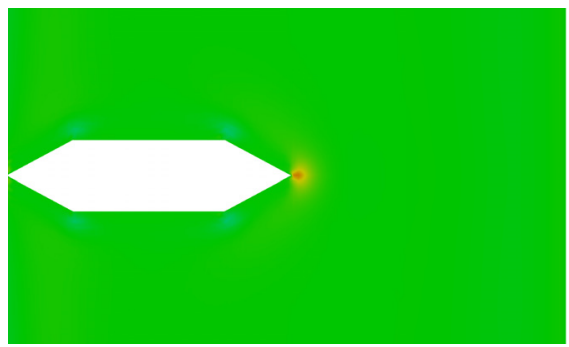

(c)

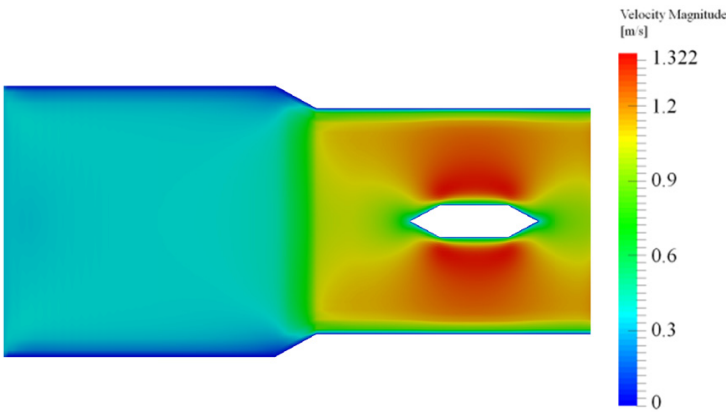

(b)

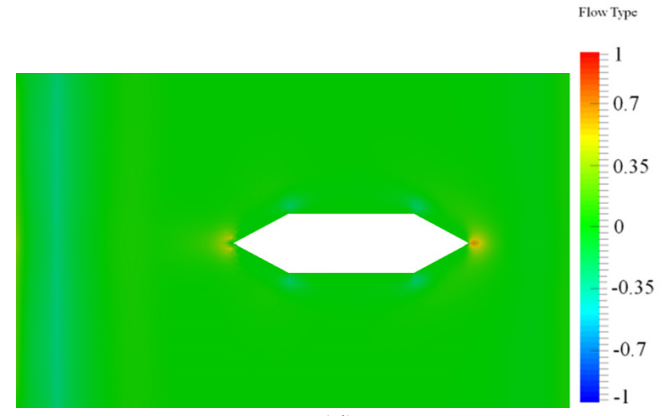

(d)

FIGURE 6. Contours of velocity magnitude (a, b) and flow type (c, d), at the middle channel height, for $d_{1}=27 \mathrm{~mm}$ (a, c), and $d_{3}=12 \mathrm{~mm}(\mathrm{~b}, \mathrm{~d})$ for an outlet average velocity of $0.423 \mathrm{~m} \cdot \mathrm{min}^{-1}\left(\alpha_{\mathrm{i}}=0.1\right)$

Regarding the experimental work, transversal samples were cut from the extruded tapes produced with the three different locations of the spider leg $\left(d_{1}, d_{2}\right.$ and $\left.d_{3}\right)$, at the two flow rates. These samples were characterized with tensile and flexural mechanical tests. The results obtained on these tests indicate that tensile and flexural strength were reduced respectively by $11 \%$ and $22 \%$ when the spider leg is moved from $d_{1}$ to $d_{3}$. When the flow rate is increased from 4 to $6.3 \mathrm{~kg} \cdot \mathrm{h}^{-1}$ the tensile and flexural strength were reduced by $41 \%$ and $36 \%$, respectively (Fig. 7). 


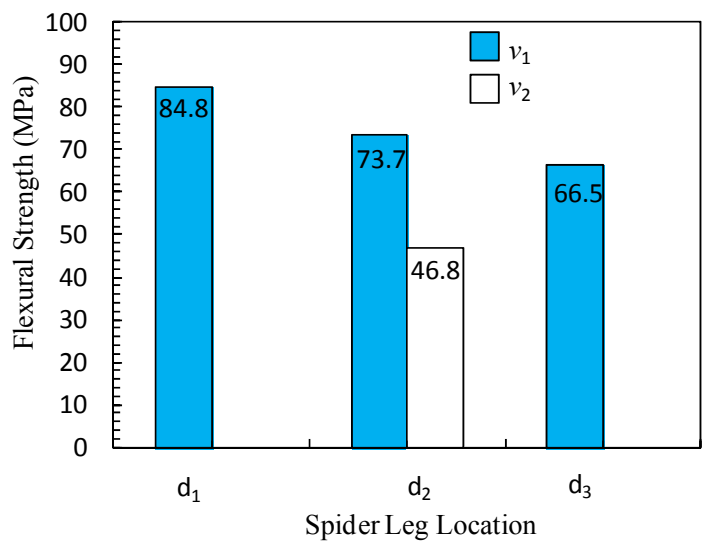

FIGURE 7. Flexural strength as function of the spider leg location, for the two different flow rates used in this study ( $v_{1}$ stands for the lower flow rate and $v_{2}$ stands for the higher flow rate).

According to Huang and Prentice [14] the fatigue life of the weld line was improved as the shear stress and shear rate at the spider rear end (where the flow fronts merge) was reduced: "These local high shear stresses, the associated extensional stresses and the subsequent orientation created by the presence of the spider, must relax so that the polymer molecules can return to their randomly oriented state...". Their conclusion leads to the idea that the weld line strength is directly related to the reduction of the stresses in the spider rear end ("a decrease in stress will allow the entanglement of the molecules"). Although Huang and Prentice mention "extensional stresses", their conclusions are based on the shear rate and shear stresses (also, they considered only inelastic fluids). Mitsuhashi et al. [15-17], improved the work of Huang and Prentice [14] by considering viscoelasticity, and showed that a reduction of the normal and shear stresses at the spider leg rear end was possible with slight changes in the shape of the spider leg. The inclusion of normal stresses and normal stress differences in their study is crucial, since the mechanism believed to be behind the formation of weld lines is based on the presence of an extensional flow, as shown in Fig. 1a.

In this work, we want to study the influence of the spider leg location and flow rate on the impact of the formed of weld lines, and therefore, based on the work of Mitsuhashi et al. [15-17], in our numerical simulations we measured the normal stress difference $\mathrm{N} 1=\tau_{\mathrm{xx}}-\tau_{\mathrm{yy}}$, from the spider rear end to the extrusion die exit (Fig. 7).

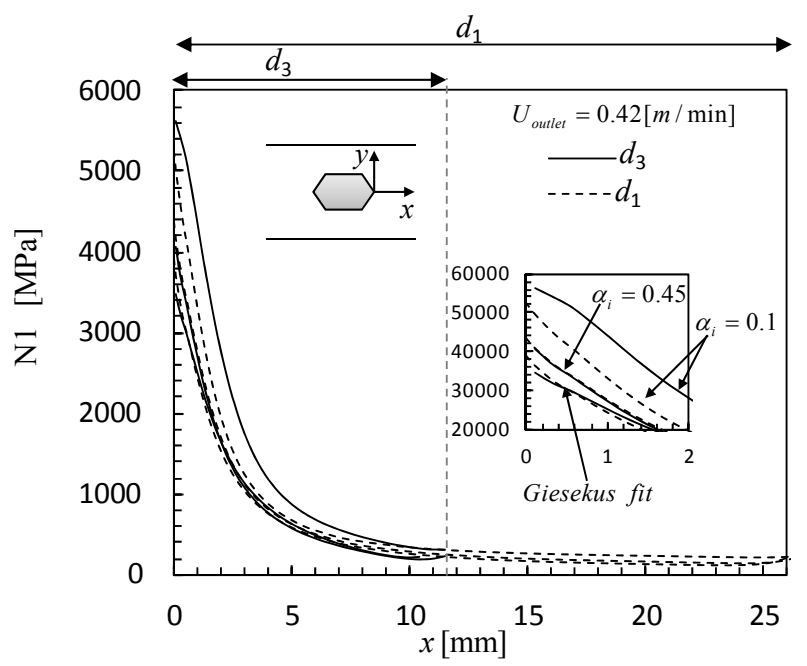

(a)

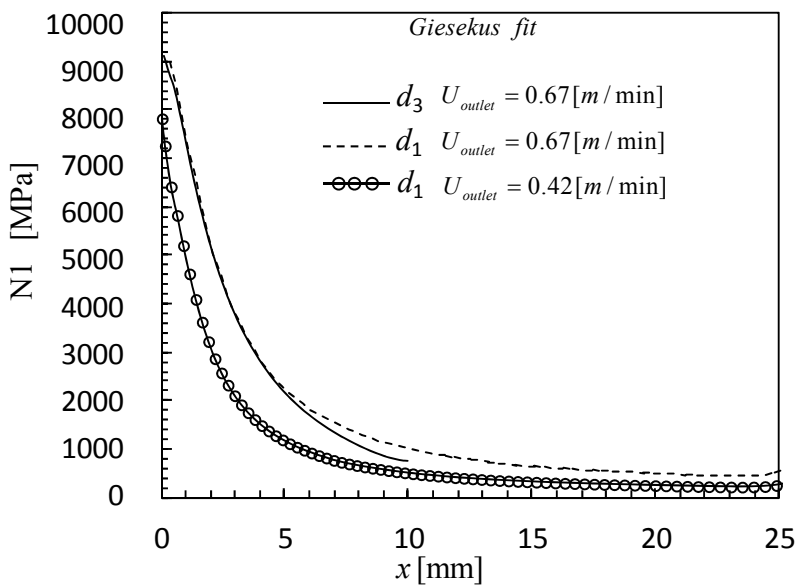

(b)

FIGURE 8. Variation of normal stress differences for: (a) different models and different locations of the spider leg; (b) different flow rate ( $U_{\text {outlet }}$ stands for the mean velocity at the die exit) and Giesekus fit. 
The evolution of $\mathrm{N} 1$ along the channel downstream the spider leg rear end shows an exponential reduction until achieving a steady state value. As expected, there is a decrease of the $N_{1}$ absolute value from $\alpha_{\mathrm{i}}=0.1$ to $\alpha_{\mathrm{i}}=0.45$ and an additional drop for the Giesekus fit (see Fig. 8a). This is a direct consequence of the material elongational viscosity (Fig. 5), that is higher when $\alpha_{\mathrm{i}}=0.1$ and lower for the Gisekus fit, independently of the elongational rate.

The distribution of N1 (Fig. 8) allows to conclude that when the spider leg is closer to the die exit the flow front interface is subjected to high average N1 values, which is known to difficult the molecular entanglement between different flow fronts [14-17]. This is in accordance with the experimental results obtained (Fig. 7).

Based on the previous results available in the literature [14-17], the average N1 value at the interface, after the flow front merging, would provide an indication of the weld line region quality. Thus, the effectiveness of the welding is expected to increase with the decrease of the average N1, and vice-versa. Figure 8 shows the average N1 values that the flow is subjected to, when moving from the spider leg rear end to the flow channel outlet, when two different spider leg locations $\left(d_{1}\right.$ and $\left.d_{3}\right)$ and two different flow rates are considered ( $v_{1}$ and $\left.v_{2}\right)$.

Based on the numerical values obtained, the ratio between the "increase of the average N1 promoted by moving the spider leg between $d_{1}$ and $d_{3}$ " and "the increase of the average N1 when the flow rate is increased from $v_{1}$ to $v_{2}$ ", is $1.21,0.79$ and 0.87 , respectively for the Giesekus fit, $\alpha_{i}=0.45$ and $\alpha_{i}=0.1$. This shows that for the Giesekus fit the decrease of the distance to the die exit has more impact on the weld line quality than the increase on flow rate, while the opposite behavior is observed for $\alpha_{\mathrm{i}}=0.45$ and $\alpha_{\mathrm{i}}=0.1$.

The experimental results obtained show a tendency that is in accordance with the trial constitutive models, $\alpha_{\mathrm{i}}=$ 0.45 and $\alpha_{\mathrm{i}}=0.1$ (since the effect of the flow rate is stronger than the effect of changing the spider leg location - see Fig. 7).

The importance of the material elongational flow on the quality of the weld at the flow front interface highlights the need of a more accurate material characterization that should include the elongational viscosity.

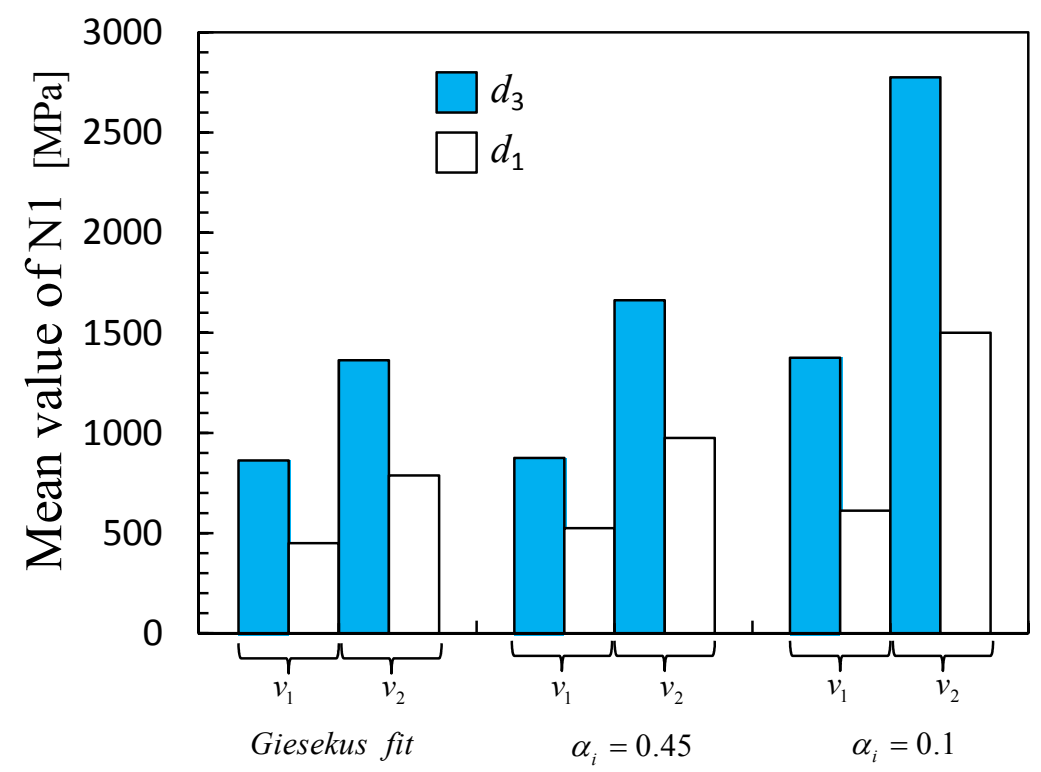

FIGURE 8. Average N1 between the spider leg rear and die outlet for the three different models used in this work $\left(\alpha_{\mathrm{i}}=0.1, \alpha_{\mathrm{i}}=\right.$ 0.45 and the Giesekus fit), distances from the merging location to the die outlet $\left(d_{1}, d_{3}\right)$ and extrusion flow rates $\left(v_{1}\right.$ and $\left.v_{2},\right)$.

\section{CONCLUSIONS}

In this work we performed experimental and numerical studies to determine the influence of the spider leg location in the weld line strength. The average first normal stress difference at flow front interface, predicted numerically, was used to evaluate the impact of the weld line on the material strength. Both numerical and experimental results revealed that a spider leg located near the die outlet promotes a lower mechanical performance of the extrudate. However, some contradictory results obtained about the relative importance of the spider leg 
location and the flow rate effects demand additional investigation on the subject and a more accurate material characterization, which should include, at least, the experimental characterization of its elongational viscosity.

\section{ACKNOWLEDGMENTS}

The authors gratefully acknowledge funding by COMPETE, FEDER and Fundação para a Ciência e a Tecnologia (FCT) through projects PEst-C/CTM/LA0025/2013 (Strategic Project - LA 25 - 2013-2014) and MATEPRO NORTE-07-0124-FEDER-000037.

\section{REFERENCES}

1. M. Z. Tadmor, J. Appl. Poly. Sci. 18, 1753-1772 (1974).

2. J. K. Kim, J. H. Song, S. T. Chung and T. H. Kwon, Polym. Eng. Sci. 37, 228-241 (1997).

3. R. Pisipati and D. G. Baird, "Correlation of non-linear rheological properties of polymer melts with weld-line strength", in Polymer processing and properties, edited by G. Astarita and L. Nicolais (Springer, USA, 1984), pp. 215-228;

4. J. M. Nóbrega, O. S. Carneiro, F. T. Pinho and P. J. Oliveira, Int. Polym. Process. 19, 225-235 (2004).

5. E. M. Hagerman, Plast. Eng. 29, 67-69 (1973).

6. P. Hubbauer, Plast. Eng. 29, 37-39 (1973).

7. P. J. Cloud, F. McDowell and S. Gerakaris, Plast. Tech. 22, 48-51 (1976).

8. S. C. Malguarnera and A. Minisali, Polym. Eng. Sci. 21, 586-593 (1981).

9. G. Prall, Mod. Plast. 47, 118 (1970).

10. S. Y. Hobbs, Polym. Eng. Sci. 14, 621-626 (1974).

11. C. Thamm, Rubber Chem. Tech. 50, 24-34 (1977).

12. S. C. Malguarnera and D. C. Riggs, Polym. Plast. Technol. Eng. 17, 193-209 (1981).

13. R. A. Worth, Polym. Eng. Sci. 20, 551-554 (1980).

14. Y. Huang, P. Prentice, Polym. Eng. Sci. 38, 1506-1522 (1998).

15. M. Mitsuhashi, K. Nishimura, K. Nomura, T. Yamamoto, N. Mori and K. Nakamura, J. Textile Eng. 47 1-8 (2001).

16. M. Mitsuhashi, K. Nishimura, K. Nomura, T. Yamamoto, N. Mori and K. Nakamura, J. Textile Eng. 48, 91101 (2002).

17. M. Mitsuhashi, K. Nishimura, K. Nomura, T. Yamamoto, N. Mori and K. Nakamura, J. Textile Eng. 49, 14-22 (2003).

18. H. Giesekus, J. Non-Newtonian Fluid Mech. 11, 69-109 (1982).

19. H. Jasak, A. Jemcov and Z. Tukovic, OpenFOAM: A C ++ Library for Complex Physics Simulations, International Workshop on Coupled Methods in Numerical Dynamics, Dubrovnik, Croatia, September (2007) pp. 1-20.

20. R. I. Issa, J. Comp. Phys. 62, 40-65 (1986).

21. O. S. Carneiro, J. M. Nóbrega, A. R. Mota and W. S. Yallew, "Prototype system for studying the effect of weld lines on extruded products" in PPS-30 Annual Meeting of the Polymer Processing Society (Cleveland, USA, June 2014).

22. C. Baiga and V. G. Mavrantzas, J. Chem. Phys. 132, 014904 (2010).

23. A. Bach, K. Almdal, H. K. Rasmussen, O. Hassager, Macromolecules 36 (14), 5174-5179 (2003).

24. J. K. Nielsen, H. K. Rasmussen, O. Hassager and G. H..McKinley, J. Rheol. 50, 453-476 (2006).

25. J. S. Lee, R. Dylla-Spears, N. P. Teclemariam and S. J. Muller, Appl. Phys. Lett. 90, 074103 (2007).

26. R. L. Thomson and P. R. S. Mendes, Int. J. Eng. Sci. 43, 79-105 (2005).

27. R. L. Thomson, Int. J. Eng. Sci. 46, 224-249 (2008). 